\title{
IMPROVING THE SPATIAL DIMENSIONALITY OF GAUSS-LEGENDRE AND EQUIANGULAR SAMPLING SCHEMES ON THE SPHERE
}

\author{
Zubair Khalid ${ }^{\dagger}$, Rodney A. Kennedy ${ }^{\star}$ and Salman Durrani ${ }^{\star}$ \\ ${ }^{\dagger}$ School of Science and Engineering, Lahore University of Management Sciences, Lahore, Pakistan \\ * Research School of Engineering, The Australian National University, Canberra, ACT 2601, Australia \\ Email: zubair.khalid@lums.edu.pk,rodney.kennedy@anu.edu.au,salman.durrani@anu.edu.au
}

\begin{abstract}
For the fast and exact computation of spherical harmonic transform (SHT) of a band-limited signal defined on the sphere from its samples, the Gauss-Legendre (GL) and equiangular sampling schemes on the sphere require asymptotically least number of samples. In comparison to the equiangular scheme, the GL scheme has larger spatial dimensionality, defined as the number of the samples required for the exact computation of SHT. In this work, we propose an efficient GL sampling scheme with spatial dimensionality equal to that of equiangular scheme. We also propose optimisation of samples along longitude to further reduce the spatial dimensionality of equiangular, GL and efficient GL sampling schemes. Furthermore, we demonstrate that the accuracy of the SHT is not affected with the proposed reduction in the spatial dimensionality.
\end{abstract}

Index Terms - spherical harmonic transform, sampling, harmonic analysis, band-limited signal, spherical harmonics.

\section{INTRODUCTION}

Signals on the sphere appear in a variety of fields of science and engineering (e.g., [1-5]). Processing of signals on the sphere in the harmonic domain is enabled by the spherical harmonic transform (SHT)-well-known counterpart of the Fourier transform. Therefore, the ability to compute SHT of a signal from its samples (or measurements) is of significant importance. Furthermore, in applications where the cost of acquiring samples on the sphere can be large (e.g., [6]), the sampling scheme on the sphere should have minimum spatial dimensionality defined as the number of samples required for the exact computation of SHT [7]. In this work, we propose optimisations to reduce the number of samples required by the existing sampling schemes that allow exact computation of SHT of band-limited signals on the sphere.

The development of sampling schemes on the sphere and methods to compute spherical harmonic transform from samples has been extensively explored in the literature, for example [7-15] and the references therein. In this work, we focus on the iso-latitude sampling schemes [7-11,13-15], where the samples along longitude are taken over iso-latitude rings, as these schemes enable efficient computation of SHT due to separation of variables. Among these schemes, the equiangular $[9,13,14]$ and Gauss-Legendre (GL) $[8,15,16]$ sampling schemes require asymptotically least number of samples for the exact computation of SHT of a band-limited signal.

For the exact computation of SHT of a signal band-limited at spherical harmonic degree $L$ (formally defined in Section 2.1),

This work is supported by the Australian Research Council's Discovery Projects funding scheme (Project no. DP150101011). the Gauss-Legendre $[15,16]$ scheme, which we refer to as standard Gauss-Legendre sampling, requires $L$ samples, chosen as the roots of Legendre polynomial of degree $L$, along co-latitude and $2 L-1$ equiangular samples along longitude in each iso-latitude ring. Thus, the spatial dimensionality of standard GL scheme is $N_{\mathrm{GL}}=L(2 L-1)$. We note that the samples along latitude in the the GL scheme are symmetric around equator. Such a symmetry of samples is useful in a sense that it reduces the computational time to evaluate SHT [15], approximately, by a factor of two. Furthermore, the symmetry of samples is required in diffusion medical resonance imaging application where the diffusion signal is antipodal in nature $[2,6]$.

The equiangular sampling on the sphere proposed in [14] requires least number of samples for the exact computation of SHT. The equiangular scheme proposed in [14] requires $L$ samples along co-latitude, where a sample is taken at either of the poles. Due to the presence of a sample at the pole, the spatial dimensionality of the equiangular scheme [14] is $N_{\mathrm{E}}=(L-1)(2 L-1)+1$, which is less than the spatial dimensionality of standard GL scheme by $N_{\mathrm{GL}}-N_{\mathrm{E}}=2(L-1)$.

In this work, we address the following questions:

- Can we design an efficient GL quadrature based sampling scheme with spatial dimensionality $N_{\mathrm{E}}$, that is, equal to the spatial dimensionality of equiangular scheme?

- Can we further improve the spatial dimensionality of both GL and equiangular sampling schemes such that they require fewer than $N_{\mathrm{E}}$ samples for the exact SHT computation?

- How do the the proposed improvements in the spatial dimensionality of different sampling schemes impact on the accuracy of the SHT?

In order to answer these questions, we organise the remainder of the paper as follows. We review the mathematical preliminaries in Section 2. An efficient GL sampling scheme on the sphere is presented in Section 3 and the optimisation of samples along longitude to reduce the spatial dimensionality is proposed in Section 4. The numerical accuracy of the SHT of a signal sampled over sampling schemes with proposed optimisations is evaluated in Section 5 and finally, the conclusions are made in Section 6.

\section{SIGNALS ON THE SPHERE}

In this work, we consider finite energy functions, referred to as signals, of the form $f(\theta, \phi)$, defined on unit sphere $\mathbb{S}^{2} \triangleq\{\mathbf{u} \in$ $\left.\mathbb{R}^{3}:|\mathbf{u}|=1\right\}$, where $\theta \in[0, \pi]$ represents the co-latitude, $\phi \in[0,2 \pi)$ represents the longitude and $|\cdot|$ denotes the Euclidian 
norm. The space of square integrable complex valued functions defined on the $\mathbb{S}^{2}$ forms a complete Hilbert space $L^{2}\left(\mathbb{S}^{2}\right)$ [17].

\subsection{Harmonic Analysis on the Sphere}

The spherical harmonic functions (or spherical harmonics) form a complete orthonormal set of basis functions of the space $L^{2}\left(\mathbb{S}^{2}\right)$ [17] and are defined for integer degree $\ell \geq 0$ and integer order $|m| \leq \ell$ as [17]

$$
Y_{\ell}^{m}(\theta, \phi) \triangleq \sqrt{\frac{2 \ell+1}{4 \pi} \frac{(\ell-m) !}{(\ell+m) !}} P_{\ell}^{m}(\cos \theta) e^{i m \phi},
$$

where $P_{\ell}^{m}(\cdot)$ represents the associated Legendre functions [17]. Due to completeness of spherical harmonics, any signal $f \in L^{2}\left(\mathbb{S}^{2}\right)$ can be expanded as

$$
f(\theta, \phi)=\sum_{\ell=0}^{\infty} \sum_{m=-\ell}^{\ell}(f)_{\ell}^{m} Y_{\ell}^{m}(\theta, \phi),
$$

where $(f)_{\ell}^{m}$ denotes the spherical harmonic coefficient of degree $\ell$ and order $m$, and is calculated using the spherical harmonic transform (SHT) given by

$$
(f)_{\ell}^{m} \triangleq \int_{\mathbb{S}^{2}} f(\theta, \phi) \overline{Y_{\ell}^{m}(\theta, \phi)} \sin \theta d \theta d \phi .
$$

The signal $f \in L^{2}\left(\mathbb{S}^{2}\right)$ is defined to be band-limited at degree $L$ if $(f)_{\ell}^{m}=0$ for $\ell \geq L$. The set of bandlimited signals forms an $L^{2}$ dimensional subspace of $L^{2}\left(\mathbb{S}^{2}\right)$, which is denoted by $\mathcal{H}_{L}$.

\subsection{Sampling Schemes on the Sphere - Notation}

We define the iso-latitude sampling on the sphere in a general setting. Let the sample positions along co-latitude are denoted by $\theta_{1}, \theta_{2}, \ldots$, where the samples are indexed, for convenience, in the increasing order of their angular distance from the equator $(\theta=\pi / 2)$. For sampling along longitude, we choose equally spaced sampling points along $\phi$ in each iso-latitude ring. Now we define the GL sampling and equiangular sampling schemes for which the SHT of a band-limited signal $f \in \mathcal{H}_{L}$ can be exactly computed.

\section{Standard Gauss-Legendre (GL) Sampling}

The standard GL scheme $[15,16]$, denoted by $\mathfrak{S}_{\mathrm{GL}}(L)$, composed of $L$ samples, chosen as the roots of Legendre polynomial of degree $L$, along co-latitude and $2 L-1$ equiangular samples along longitude in each iso-latitude ring.

\section{Equiangular Sampling on the Sphere}

Here, we consider a variant of the equiangular scheme [14], denoted by $\mathfrak{S}_{\mathrm{E}}(L)$, composed of $L+1$ equiangular samples along co-latitude with samples at both of the poles and $2 L-1$ equiangular samples along longitude in each iso-latitude ring. Due to the placement of samples at both of the poles, the spatial dimensionality of $\mathfrak{S}_{\mathrm{E}}(L)$ is $N_{\mathrm{E}}+1$.

\section{EFFICIENT GAUSS-LEGENDRE SCHEME}

In this section, we address the first question posed in the Section 1 and propose the GL quadrature based sampling scheme of spatial dimensionality $N_{\mathrm{E}}$.

\subsection{Harmonic Formulation}

For a band-limited signal $f \in \mathcal{H}_{L}$, the summation over degree $\ell$ in (2) is truncated at $L-1$. By defining

$$
G_{m}(\theta)=\sum_{\ell=|m|}^{L-1}(f)_{\ell}^{m} \tilde{P}_{\ell}^{m}(\theta)
$$

where $\tilde{P}_{\ell}^{m}(\theta) \triangleq Y_{\ell}^{m}(\theta, 0)$ denotes scaled associated Legendre functions, we rewrite the spherical harmonic expansion, given in (2), of a band-limited signal $f \in \mathcal{H}_{L}$ as

$$
f(\theta, \phi)=\sum_{m=-(L-1)}^{L-1} G_{m}(\theta) e^{i m \phi},
$$

which can be substituted in (3) to reformulate the SHT as

$$
(f)_{\ell}^{m}=2 \pi \int_{\theta=0}^{\pi} G_{m}(\theta) \tilde{P}_{\ell}^{m}(\theta) d \theta
$$

where the orthogonality of complex exponentials is employed.

\subsection{Efficient Gauss-Legendre Sampling on the Sphere}

We propose an efficient GL sampling on the sphere, denoted by $\mathfrak{S}_{\mathrm{EGL}}(L)$, composed of $L-1$ iso-latitude rings of samples placed at $\theta_{1}, \theta_{2}, \ldots, \theta_{L-1}$ taken as roots of Legendre polynomial of degree $L-1, P_{L-1}^{0}$, and an additional sample $\theta_{L}$ at either of the poles. Similar to the standard GL scheme and equiangular scheme, we take $2 L-1$ number of samples along longitude in each iso-latitude ring.

Remark 1 The proposed efficient GL scheme $\mathfrak{S}_{\mathrm{EGL}}(L)$ has spatial dimensionality $N_{E G L}=(L-1)(2 L-1)+1=N_{E}$, that is, $\mathfrak{S}_{\mathrm{EGL}}(L)$ requires $2 L-2$ fewer samples than required by the standard $G L$ scheme $\mathfrak{S}_{\mathrm{GL}}(L)$ for SHT computation.

We now show that the SHT of a band-limited signal can be computed by taking the samples of the signal over $\mathfrak{S}_{\mathrm{EGL}}(L)$. The formulation of a signal $f$ in (5) indicates that $G_{m}\left(\theta_{r}\right)$, for each $\theta_{r}$ and each $|m|<L$, can be computed by taking FFT over $2 L-1$ samples along an iso-latitude ring placed at $\theta_{r}$. Once $G_{m}\left(\theta_{r}\right)$ is computed for each $|m|<L$ and each $\theta_{r}$, the spherical harmonic coefficients $(f)_{\ell}^{m}$ can be computed using (6) provided the integral involved can be evaluated exactly over the number of samples along co-latitude.

Using (4), we rewrite the integrand in (6) as

$$
I_{\ell}^{m}(\theta)=G_{m}(\theta) \tilde{P}_{\ell}^{m}(\theta)=\sum_{\ell^{\prime}=|m|}^{L-1}(f)_{\ell^{\prime}}^{m} \tilde{P}_{\ell^{\prime}}^{m}(\theta) \tilde{P}_{\ell}^{m}(\theta) .
$$

For $\ell<L-1$, the polynomial degree of the integrand $I_{\ell}^{m}(\theta)$ is $L+\ell-1$ or the maximum polynomial degree is $2 L-3$. If we take $L-1$ samples along co-latitude as roots of Legendre polynomial $P_{L-1}^{0}$, the Gauss-Legendre quadrature allows to compute the integral exactly if the degree of integrand is less than $2 L-3$. Hence, the spherical harmonic coefficients $(f)_{\ell}^{m}$ of all degrees $\ell<L-1$ and orders $|m| \leq \ell$ can be computed by discretising the integral in (6) as

$$
(f)_{\ell}^{m}=2 \pi \sum_{r=1}^{L-1} G_{m}\left(\theta_{r}\right) \tilde{P}_{\ell}^{m}\left(\theta_{r}\right) q\left(\theta_{r}\right) \quad \ell \leq L-1,
$$


where $q\left(\theta_{r}\right)$ denotes the GL quadrature weight given by

$$
q\left(\theta_{r}\right)=\frac{2 L-1}{2 \pi}\left(\frac{\sin \theta_{r}}{(L-1) P_{L-2}^{0}\left(\theta_{r}\right)}\right)^{2} .
$$

We yet need to compute the spherical harmonic coefficients of degree $L-1$, that is, $(f)_{L-1}^{m}$ for $|m|<L$. We first focus on the computation of $(f)_{L-1}^{m}$ for $m \neq 0$. For $\ell=L-1$ and $m \neq 0$, the maximum polynomial degree of the integrand $I_{\ell}^{m}(\theta)$ is $2 L-2$, due to which the GL quadrature rule with $L-1$ samples, that supports the computation of integral of degree $2 L-3$, may not be directly applied. However, the GL quadrature rule with $L-1$ samples along colatitude ensures that the orthogonality between $\tilde{P}_{L-1}^{m}(\theta)$ and $\tilde{P}_{\ell^{\prime}}^{m}(\theta)$ for $\ell^{\prime}<L-1$ is preserved as the product $\tilde{P}_{\ell^{\prime}}^{m}(\theta) \tilde{P}_{L-1}^{m}(\theta)$ always have the maximum degree of $2 L-3$. Thus, $(f)_{L-1}^{m}$ for $m \neq 0$ can be computed as follows:

$$
(f)_{L-1}^{m}=\frac{2 \pi}{Q_{L}^{m}} \sum_{r=1}^{L-1} G_{m}\left(\theta_{r}\right) \tilde{P}_{L-1}^{m}\left(\theta_{r}\right) q\left(\theta_{r}\right) \quad m \neq 0,
$$

where

$$
Q_{L}^{m}=\sum_{r=1}^{L-1}\left(\tilde{P}_{L-1}^{m}\left(\theta_{r}\right)\right)^{2} q\left(\theta_{r}\right)
$$

serves as a normalization constant.

The formulation in (10) cannot be used for the computation of $(f)_{L-1}^{0}$ as the samples $\theta_{r}, r \in[1, L-1]$ are chosen as the roots of $P_{L-1}^{0}$, due to which there will be no contribution of $(f)_{L-1}^{0}$ towards $G_{0}\left(\theta_{r}\right)$. In order to compute $(f)_{L-1}^{0}$, we use the signal at $L$-th sample taken at either of the poles, that is, $\theta_{L}=0$ or $\theta_{L}=\pi$. Since we have already computed $(f)_{\ell}^{0}$ for $0 \leq \ell<L-1,(f)_{L-1}^{0}$ can be computed using (4) as

$$
(f)_{L-1}^{0}=\frac{f\left(\theta_{L}, 0\right)-\sum_{\ell=0}^{L-2}(f)_{\ell}^{0} \tilde{P}_{\ell}^{0}\left(\theta_{L}\right)}{P_{L-1}^{0}\left(\theta_{L}\right)},
$$

where we have noted $G_{0}\left(\theta_{L}\right)=f\left(\theta_{L}, 0\right)$. It is worth noting here that $P_{L-1}^{0}\left(\theta_{L}\right) \neq 0$ for $\theta_{L}=0, \pi$ and any band-limit $L$.

\section{OPTIMISATION OF SAMPLES ALONG LONGITUDE}

With an objective to reduce the total number of samples required for the computation of SHT, we here present an optimisation to reduce the number of samples taken along longitude in the iso-latitude rings. The proposed optimisation can be applied to either standard GL sampling $\mathfrak{S}_{\mathrm{GL}}(L)$, efficient GL sampling $\mathfrak{S}_{\mathrm{EGL}}(L)$ or equiangular sampling $\mathfrak{S}_{\mathrm{E}}(L)$.

\subsection{Optimisation of samples}

We first define the optimisation of samples along longitude and later we show that the SHT of a band-limited signal can be computed exactly from its values taken over the proposed sampling scheme. Instead of taking $2 L-1$ samples along each iso-latitude ring as required by $\mathfrak{S}_{\mathrm{GL}}(L), \mathfrak{S}_{\mathrm{EGL}}(L)$ or $\mathfrak{S}_{\mathrm{E}}(L)$, we propose to choose $2 L-1$ equiangular samples in the ring placed at $\theta_{1}, 2 L-3$ equiangular samples in the ring placed at $\theta_{2}$ and $2 L-5$ equiangular samples in the remaining iso-latitude rings. We add a superscript ' $O$ ' to the notation $\mathfrak{S}_{\mathrm{GL}}(L), \mathfrak{S}_{\mathrm{EGL}}(L)$ or $\mathfrak{S}_{\mathrm{E}}(L)$ to denote this optimisation (or reduction) of samples along longitude and the sampling schemes are referred to as optimised sampling schemes.

\subsection{Computation of SHT}

For each sampling scheme, the computation of SHT requires to compute $G_{m}\left(\theta_{r}\right)$ for each order $|m|<L$ and each sample $\theta_{r}$ along colatitude. In order to enable this computation, these schemes consider $2 L-1$ samples along each iso-latitude ring. We here show that $G_{m}$ can be computed for each $|m|<L$ and each sample $\theta_{r}$ for each optimised sampling scheme $\left(\mathfrak{S}_{\mathrm{GL}}^{\mathrm{O}}(L), \mathfrak{S}_{\mathrm{EGL}}^{\mathrm{O}}(L)\right.$ or $\left.\mathfrak{S}_{\mathrm{E}}^{\mathrm{O}}(L)\right)$.

Since we have taken $2 L-1$ number of samples in a ring placed at $\theta_{1}$ (closest to the equator $\left.\theta=\pi / 2\right), G_{m}\left(\theta_{1}\right)$ for each $|m|<L$ can be computed by employing FFT following (5). As there is only one coefficient of each order $L-1$ and $-(L-1),(f)_{L-1}^{L-1}$ and $(f)_{L-1}^{-(L-1)}$ computed from $G_{L-1}\left(\theta_{1}\right)$ and $G_{-(L-1)}\left(\theta_{1}\right)$, respectively, using (4) as

$$
(f)_{L-1}^{m} \triangleq \frac{G_{m}\left(\theta_{1}\right)}{\tilde{P}_{L-1}^{m}\left(\theta_{1}\right)}, \quad|m|=L-1, \theta_{1} \neq 0, \pi .
$$

Note that the denominator $\tilde{P}_{L-1}^{m}\left(\theta_{1}\right) \propto\left(\sin \theta_{1}\right)^{m}$ is always nonzero for $|m|=L-1, \theta_{1} \neq 0, \pi$.

Once we compute $(f)_{L-1}^{m}$ for $|m|=L-1$, these can be used to update the signal at all sample positions (except for the samples in the ring placed at $\theta_{1}$ ) as follows:

$$
f(\theta, \phi) \leftarrow f(\theta, \phi)-\tilde{f}_{L-1}(\theta, \phi)
$$

where

$$
\tilde{f}_{m}(\theta, \phi) \triangleq \sum_{\ell=m}^{L-1}\left((f)_{\ell}^{m} Y_{\ell}^{m}(\theta, \phi)+(f)_{\ell}^{-m} Y_{\ell}^{-m}(\theta, \phi)\right)
$$

After the update in (14), the signal $f$, given in (5), at the remaining samples can be reformulated as $f(\theta, \phi)=\sum_{m=-(L-2)}^{L-2} G_{m}(\theta) e^{i m \phi}$, which indicates that the signal now does not have a contribution of complex exponentials $e^{i(L-1) \phi}$ and $e^{-i(L-1) \phi}$. Thus, we only need $2 L-3$ number of samples in the ring placed at $\theta_{2}$ in order to recover $G_{m}\left(\theta_{2}\right)$ for $|m|<L-1$ using FFT. Now we have $G_{m}\left(\theta_{1}\right)$ and $G_{m}\left(\theta_{2}\right)$ for all $|m|<L$, which can be used to compute the spherical harmonic coefficients $(f)_{\ell}^{m}$ of order $|m|=L-2$ and degree $\ell=$ $L-2, L-1$. The location of $\theta_{1}$ depends on the band-limit $L$ and the choice of sampling scheme, due to which the computation of $(f)_{\ell}^{m}$ of order $|m|=L-2$ is presented for two cases. We first note that $\tilde{P}_{L-2}^{m}(\theta) \propto(\sin \theta)^{m}$ and $\tilde{P}_{L-1}^{m}(\theta) \propto(\sin \theta)^{m} \cos \theta$ for $|m|=L-2[17]$.

Case 1: $\theta_{1}=\pi / 2$

This case arises when the band-limit $L$ is odd for $\mathfrak{S}_{\mathrm{GL}}(L)$ and even for $\mathfrak{S}_{\mathrm{EGL}}(L)$ and $\mathfrak{S}_{\mathrm{E}}(L)$. We consider $|m|=L-2$ throughout this subsection. Due to the presence of $\cos \theta$ in $\tilde{P}_{L-1}^{m}(\theta)$, the spherical harmonic coefficient $(f)_{L-1}^{m}$ does not contribute to $G_{m}\left(\theta_{1}\right)$. Therefore, $(f)_{L-2}^{m}$ can be computed using $G_{m}\left(\theta_{1}\right)$ as follows

$$
(f)_{L-2}^{m} \triangleq \frac{G_{m}\left(\theta_{1}\right)}{\tilde{P}_{L-2}^{m}\left(\theta_{1}\right)}, \quad|m|=L-2, \theta_{1} \neq 0, \pi,
$$

which can be used to determine $(f)_{L-1}^{m}$ using $G_{m}\left(\theta_{2}\right)$ through

$$
(f)_{L-1}^{m} \triangleq \frac{G_{m}\left(\theta_{2}\right)-(f)_{L-2}^{m} \tilde{P}_{L-2}^{m}\left(\theta_{2}\right)}{\tilde{P}_{L-1}^{m}\left(\theta_{2}\right)}, \quad|m|=L-2 .
$$




\section{Case 2: $\theta_{1} \neq \pi / 2$}

For this case, $\theta_{2}=\pi-\theta_{1}$, that is, symmetric around equator. The coefficients $(f)_{L-2}^{m}$ and $(f)_{L-1}^{m}$ for $|m|=L-2$ can be computed exactly using $G_{m}\left(\theta_{1}\right)$ and $G_{m}\left(\theta_{2}\right)$ by setting up an orthogonal system of linear equations using (4).

Once the spherical harmonic coefficients of order $|m|=L-2$ are computed, the signal at the sample positions (except for the samples in the rings placed at $\left.\theta_{1}, \theta_{2}\right)$ is updated as follows $f(\theta, \phi) \leftarrow$ $f(\theta, \phi)-\tilde{f}_{L-2}(\theta, \phi)$. With this update, the signal now have the contribution of complex exponentials $e^{i m \phi}$ for $|m|<L-2$, which implies that we only require $2 L-5$ samples in the rings placed at $\theta_{r}, r>2$ to determine $G_{m}\left(\theta_{r}\right)$ for $|m|<L-2$ using FFT.

Remark 2 With the proposed optimisation of samples along longitude, the SHT can be computed with $4 L-10, L>2$ fewer samples for efficient Gauss-Legendre scheme and equiangular scheme and $4 L-6, L>1$ fewer samples for standard Gauss-Legendre scheme. This reduction in the spatial dimensionality can be useful in applications where the band-limit of the signal is small. For example, we expect that the reduction achieved by the proposed optimisation can be of great use in diffusion magnetic resonance imaging (dMRI) application [2,6] where the band-limit is of the order $10-10^{2}$ and the cost of acquisition is proportional to the number of samples (measurements).

\section{ACCURACY ANALYSIS}

We analyse the accuracy of the SHT of a band-limited signal sampled over standard GL $\mathfrak{S}_{\mathrm{GL}}(L)$, equiangular $\mathfrak{S}_{\mathrm{E}}(L)$, efficient GL $\mathfrak{S}_{\mathrm{EGL}}(L)$ sampling schemes and optimised versions of these schemes, $\mathfrak{S}_{\mathrm{GL}}^{\mathrm{O}}(L), \mathfrak{S}_{\mathrm{E}}^{\mathrm{O}}(L)$ and $\mathfrak{S}_{\mathrm{EGL}}^{\mathrm{O}}(L)$. We implement the SHT associated with each of the sampling scheme in MATLAB in double precision arithmetic. In our implementation of SHT associated with the GL quadrature based sampling schemes, we use the three term recurrence relation [7] for the computation of scaled associated Legendre functions $\tilde{P}_{\ell}^{m}\left(\theta_{r}\right)=Y_{\ell}^{m}\left(\theta_{r}, 0\right)$ for all degrees $\ell<L$ and orders $|m| \leq \ell$ and for the required samples $\theta_{r}$ along latitude. In the implementation of SHT for equiangular sampling scheme [14], Wigner- $d$ functions are computed using the recursion relations proposed in [18].

For each sampling scheme, we carry out the accuracy test composed of following steps: 1) obtain a band-limited test signal $f_{\mathrm{T}} \in$ $\mathcal{H}_{L}$ in the harmonic domain by generating its spherical harmonic coefficients $\left(f_{\mathrm{t}}\right)_{\ell}^{m}$ for $0<\ell<L,|m| \leq \ell$ with real and imaginary parts uniformly distributed in the interval $[-1,1], 2)$ obtain the signal $f$ in the spatial domain over the samples of each sampling scheme using (2), and 3) apply SHT associated with each of the sampling scheme to the signal in the spatial domain to compute the reconstructed spherical harmonic coefficients, denoted by $\left(f_{\mathrm{r}}\right)_{\ell}^{m}$. We repeat the test 50 times and record the average value for the maximum error $E_{\max }$ given by

$$
E_{\max } \triangleq \max \left|\left(f_{\mathrm{t}}\right)_{\ell}^{m}-\left(f_{\mathrm{r}}\right)_{\ell}^{m}\right|,
$$

which is plotted in Fig. 1 for standard GL sampling $\mathfrak{S}_{\mathrm{GL}}(L)$, efficient GL sampling $\mathfrak{S}_{\mathrm{EGL}}(L)$, equiangular sampling $\mathfrak{S}_{\mathrm{E}}(L)$ and optimised versions of these schemes and band-limits in the range $16 \leq L \leq 1024$. It is evident that the reconstruction errors do not change with the proposed optimisation of samples along longitude. It can also be observed that the maximum error $E_{\max }$ is slightly larger for efficient GL scheme, which is due the normalization by the factor $Q_{L}^{m}$ in (10) for the computation of spherical harmonic coefficients of degree $L-1$.

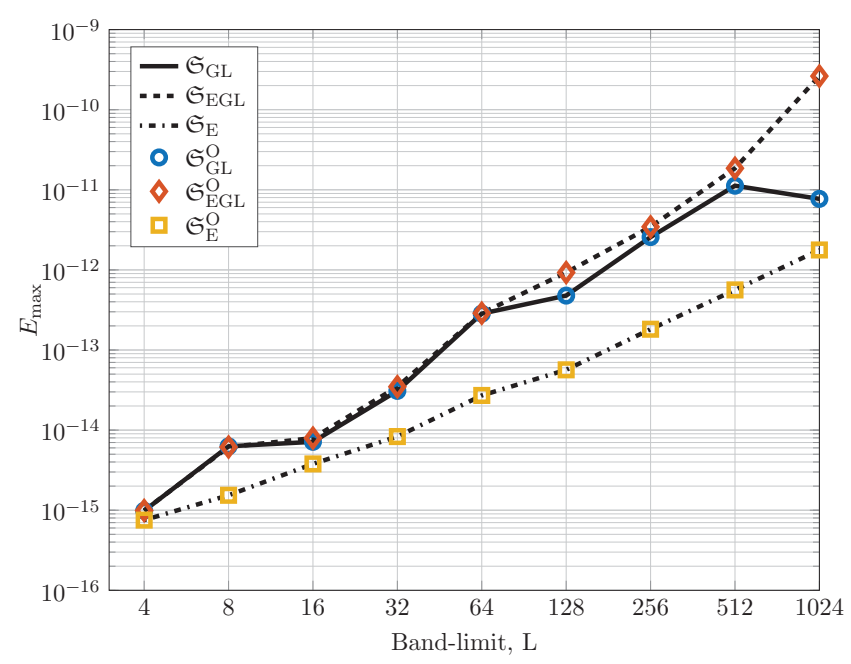

Fig. 1: The maximum error $E_{\max }$ given in (17) is plotted for different sampling schemes and band-limits in the range $16 \leq L \leq 1024$. It can be observed that the errors do not change due to the proposed optimisation of samples along longitude (indicated by the superscript 'O').

\section{CONCLUSIONS}

For the fast and exact computation of spherical harmonic transform (SHT) from the samples of a signal defined on the sphere and band-limited at spherical harmonic degree $L$, we have proposed an efficient GL sampling scheme with spatial dimensionality $N_{\mathrm{EGL}}=(L-1)(2 L-1)+1=N_{\mathrm{E}}$, that is, equal to that of equiangular scheme. For each equiangular, GL or efficient GL sampling scheme, we have also proposed the optimisation of samples along longitude to reduce the spatial dimensionality by approximately $4 L$. This reduction in the number of samples can be useful in diffusion magnetic resonance imaging (dMRI) application where the band-limit is of the order $10-10^{2}$.

\section{REFERENCES}

[1] D. N. Spergel, R. Bean, O. Doré, M. R. Nolta, C. L. Bennett, J. Dunkley, G. Hinshaw, N. Jarosik, E. Komatsu, L. Page, H. V. Peiris, L. Verde, M. Halpern, R. S. Hill, A. Kogut, M. Limon, S. S. Meyer, N. Odegard, G. S. Tucker, J. L. Weiland, E. Wollack, and E. L. Wright, "Three-year Wilkinson Microwave Anisotropy Probe (WMAP) observations: Implications for cosmology," The Astrophysical Journal Supplement Series, vol. 170, no. 2, pp. 377-408, 2007.

[2] A. P. Bates, Z. Khalid, and R. A. Kennedy, "An optimal dimensionality sampling scheme on the sphere for antipodal signals in diffusion magnetic resonance imaging," in Proc. IEEE Int. Conf. Acoust., Speech, Signal Process., ICASSP'2015, Brisbane, Austalia, Apr. 2015.

[3] N. Jarosik, C. L. Bennett, J. Dunkley, B. Gold, M. R. Greason, M. Halpern, R. S. Hill, G. Hinshaw, A. Kogut, E. Komatsu, D. Larson, M. Limon, S. S. Meyer, M. R. Nolta, N. Odegard, L. Page, K. M. Smith, D. N. Spergel, G. S. Tucker, J. L. Weiland, E. Wollack, and E. L. Wright, "Seven-year Wilkinson Microwave Anisotropy Probe (WMAP) observations: Sky maps, 
systematic errors, and basic results," Astrophys. J., vol. 192, no. 2, pp. 1-14, 2011.

[4] M. A. Wieczorek and F. J. Simons, "Minimum variance multitaper spectral estimation on the sphere," J. Fourier Anal. Appl., vol. 13, no. 6, pp. 665-692, 2007.

[5] T. S. Pollock, T. D. Abhayapala, and R. A. Kennedy, "Introducing space into MIMO capacity calculations," J. Telecommun. Syst., vol. 24, no. 2, pp. 415-436, Oct. 2003.

[6] D. S. Tuch, "Q-ball imaging," Magn. Reson. Med., vol. 52, no. 6, pp. 1358-1372, Dec. 2004.

[7] Z. Khalid, R. A. Kennedy, and J. D. McEwen, "An optimaldimensionality sampling scheme on the sphere with fast spherical harmonic transforms," IEEE Trans. Signal Process., vol. 62, no. 17, pp. 4597-4610, Sep. 2014.

[8] W. Skukowsky, "A quadrature formula over the sphere with application to high resolution spherical harmonic analysis," $J$. Geodesy, vol. 60, no. 1, pp. 1-14, Mar. 1986.

[9] J. R. Driscoll and D. M. Healy, Jr., "Computing Fourier transforms and convolutions on the 2-sphere," Adv. Appl. Math., vol. 15, no. 2, pp. 202-250, Jun. 1994.

[10] R. G. Crittenden and N. G. Turok, "Exactly azimuthal pixelizations of the sky," Arxiv preprint astro-ph/9806374, 1998.

[11] K. M. Górski, E. Hivon, A. J. Banday, B. D. Wandelt, F. K. Hansen, M. Reinecke, and M. Bartelmann, "HEALPix: A Framework for High-Resolution Discretization and Fast Analysis of Data Distributed on the Sphere," Astrophys. J., vol. 622, pp. 759-771, Apr. 2005.

[12] Y. Wiaux, L. Jacques, and P. Vandergheynst, "Fast spin 2 spherical harmonics transforms and application in cosmology," $J$. Comput, Phys., vol. 226, no. 2, pp. 2359-2371, 2005.

[13] K. M. Huffenberger and B. D. Wandelt, "Fast and exact spin-s spherical harmonic transforms," Astrophys. J. Supp., vol. 189, no. 2, pp. 255-260, Aug. 2010.

[14] J. D. McEwen and Y. Wiaux, "A novel sampling theorem on the sphere," IEEE Trans. Signal Process., vol. 59, no. 12, pp. 5876-5887, Dec. 2011.

[15] N. Schaeffer, "Efficient spherical harmonic transforms aimed at pseudospectral numerical simulations," Geochemistry, Geophysics, Geosystems, vol. 14, no. 3, pp. 751-758, Mar. 2013.

[16] A. G. Doroshkevich, P. D. Naselsky, O. V. Verkhodanov, D. I. Novikov, V. I. Turchaninov, I. D. Novikov, P. R. Christensen, and Chiang, "Gauss Legendre Sky Pixelization (GLESP) for CMB maps," Int. J. Mod. Phys. D., vol. 14, no. 02, pp. 275290, Feb. 2005.

[17] R. A. Kennedy and P. Sadeghi, Hilbert Space Methods in Signal Processing. Cambridge, UK: Cambridge University Press, Mar. 2013.

[18] S. Trapani and J. Navaza, "Calculation of spherical harmonics and Wigner $d$ functions by FFT. Applications to fast rotational matching in molecular replacement and implementation into AMoRe," Acta Cryst. A, vol. 62, no. 4, pp. 262-269, Jul. 2006. 